La syntaxe des artefacts : lier entre elles différentes logiques d'action pour expliquer les pratiques découlant du changement technologique

\title{
Carole Groleau
}

\section{OpenEdition}

\section{Journals}

Édition électronique

URL : http://journals.openedition.org/communicationorganisation/413

DOI : $10.4000 /$ communicationorganisation. 413

ISSN : 1775-3546

Éditeur

Presses universitaires de Bordeaux

Édition imprimée

Date de publication : 1 juin 2008

Pagination : $18-28$

ISBN : 978-2-86781-506-5

ISSN : $1168-5549$

Référence électronique

Carole Groleau, « La syntaxe des artefacts : lier entre elles différentes logiques d'action pour expliquer les pratiques découlant du changement technologique », Communication et organisation [En ligne], 33 | 2008, mis en ligne le 01 juin 2011, consulté le 03 mai 2019. URL : http://journals.openedition.org/ communicationorganisation/413 ; DOI : 10.4000/communicationorganisation.413 
Dossier : Conduire le changement organisationnel?

\title{
Résumé
}

Plusieurs des nouvelles approches en communication organisationnelle, inspirées des travaux de Giddens et Latour, conceptualisent les organisations comme des processus dynamiques soutenus par les interactions de leurs membres. Elles proposent aussi des avenues de recherche permettant d'appréhender les technologies au sein des collectifs. Après avoir exploré, les apports et les limites des conceptualisations formulées par ces approches, nous nous tournerons vers la cognition distribuée pour y repenser la place des technologies dans les organisations en transformation, à partir du concept de syntaxe des artefacts. Notre recherche s'appuie sur une étude de cas relatant l'expérience d'informatisation du service de comptabilité d'un centre hospitalier.

\section{Mots-clés}

Cognition distribuée, changement technologique, syntaxe des artefacts, pratiques de travail, constructivisme

\begin{abstract}
Many recent approaches in organizational communication, inspired by the works of Giddens and Latour, see organizations as constituted through interactions. These approaches also offer pertinent research avenues to conceptualize technologies within organizations. After analyzing their contributions and limits, we turn to distributed cognition using the concept of artifact syntax to reframe the role of technology in evolving organizations. To do so, we rely on a case study describing the computerization experience of accounting clerks working in a hospital.
\end{abstract}

\section{Key-Words}

Distributed cognition, technological change, artifact syntax, work practices, constructivism

\section{Carole GROLEAU}

Carole Groleau est professeure agrégée au département de communication de l'Université de Montréal. Ses recherches portent sur la place des technologies en milieu organisationnel. Plus particulièrement, elle s'est intéressée à la dimension organisante de la technologie et aux dynamiques interactionnelles soutenant le processus de changement technologique à partir d'approches comme l'action située, la cognition distribuée, la théorie de l'activité et la structuration. 


\section{La syntaxe des artefacts : lier entre elles différentes logiques d'action pour expliquer les pratiques découlant du changement technologique}

Carole Groleau

carole.groleau@umontreal.ca

Les cadres théoriques auxquels font appel plusieurs chercheurs en communication organisationnelle depuis des décennies (Putnam \& Pacanowski, 1983 ; Taylor \& Van Every, 2000) nous permettent de penser les organisations comme des processus dynamiques soutenus par les interactions de leurs membres ${ }^{1}$. Parmi ces approches, la structuration (Giddens, 1984) et les travaux de Latour (1989) proposent des avenues de recherche permettant d'appréhender les technologies au sein des collectifs. Dans un premier temps, nous explorerons les apports et les limites des conceptualisations formulées par ces chercheurs, puis, dans un second temps, nous nous tournerons vers la cognition distribuée pour repenser la place des technologies dans les organisations en transformation.

\section{La conceptualisation de la technologie : contributions et limites}

Parmi la famille des structurationnistes ${ }^{2}$, Orlikowski, qui s'est penchée sur la place des technologies dans les organisations, propose des pistes intéressantes. Elle met en parallèle la dualité du structurel de Giddens et la dualité de la technologie. Ce faisant, elle reconnait la technologie comme étant, à l'instar du structurel, un construit social qui est à la fois le produit et le médium de l'action humaine. La technologie résulte certes du travail de ses concepteurs, mais elle est aussi appropriée par des individus qui en déterminent l'usage. Dans ses travaux ultérieurs, elle s'intéresse à la problématique de

\footnotetext{
${ }^{1}$ Je remercie Nicole Giroux, Christiane Demers et Catherine Zekri pour leurs conseils. Cette recherche a reçu l'aide du Conseil de recherche en sciences humaines du Canada (CRSH 410-2004-1091).

${ }^{2}$ Pour une analyse plus poussée de ces auteurs, voir Groleau (2002).
} 
Dossier : Conduire le changement organisationnel?

l'appropriation et, plus particulièrement, aux patterns d'interaction qui émanent de l'actualisation d'habiletés et de contraintes associées à la technologie par les membres de différents collectifs (Orlikowski, $1992 ; 1996 ; 2000)$.

Du côté des travaux de Latour, nous retenons une piste qui, bien que peu explorée à date, nous semble très pertinente pour penser la spécificité des entités matérielles, telle la technologie, au sein des collectifs. En s'appuyant sur une comparaison entre le lien social de communautés de babouins et celui propre aux communautés d'humains, Strum et Latour (1987) expliquent la transcendance du lien social humain par la présence d'entités matérielles, lesquelles transmettent une vision du monde découlant de négociations et d'associations passées.

Bien que les recherches s'inspirant de Giddens et Latour reposent sur des prémisses différentes ${ }^{3}$, on voit se profiler dans ces textes des similitudes dans la manière dont est appréhendée la technologie. Plus spécifiquement, la technologie y est pensée comme un construit social formé à partir de l'expérience d'humains tant au moment de son élaboration qu'à celui de son déploiement.

Cette manière de penser la technologie fait écho aux travaux d'autres chercheurs. Par exemple, Simondon soutient que la technologie intègre l'expérience humaine : «ce qui réside dans les machines, c'est de la réalité humaine, du geste humain fixé et cristallisé en structures qui fonctionnent » $(1969$, p.12) Ici, ce ne sont pas les liens sociaux, mais le geste et la méthode humaine qui sont inscrits dans l'objet et rendus disponibles par lui.

Bien que, dans l'ensemble, ces auteurs constatent que les technologies sont des construits intégrant différentes dimensions de l'expérience humaine, peu d'entre eux proposent des concepts pouvant mieux décrire comment s'inscrit dans ces entités matérielles le vécu de générations d'individus. Afin de pallier cette lacune et de poursuivre notre réflexion sur le changement technologique, nous emprunterons à la cognition distribuée le concept de syntaxe des artefacts.

\footnotetext{
${ }^{3}$ Pour une analyse comparative de ces approches, voir le Scandinavian Journal of Information Systems (2005, 17(1)).
} 


\section{La cognition distribuée : la syntaxe des artefacts pour explorer les technologies au sein des organisations}

Les chercheurs associés à la cognition distribuée définissent la cognition comme un phénomène qui existe au-delà des frontières du corps humain. On y perçoit l'action humaine comme reposant sur la capacité de l'être humain à intégrer les éléments du contexte formé d'un ensemble d'entités, d'origine matérielle et sociale, où l'individu puise l'information nécessaire à la conduite de ses activités.

Hutchins (1995) a contribué, de manière importante, au développement de cette approche, par son étude sur l'évolution des artefacts utilisés pour soutenir le travail des marins. Selon lui, les artefacts cumulent le savoir de générations successives d'êtres humains qui les manipulent et participent à leur évolution. Il conçoit les artefacts comme des entrepôts de connaissances prenant la forme d'entités durables dans lesquelles s'inscrivent une vision du monde : «Les régularités des relations liant les différentes entités dans le monde sont construites à même la structure de l'artefact ${ }^{4} \gg(1995$, p.107). Dans le cadre de son étude sur la marine, ces régularités, qui s'organisent selon une configuration particulière qu'il nomme syntaxe, proviennent des cycles récursifs des marées et des astres, exprimés d'abord par des icônes puis, ensuite, par des codes alphanumériques, tels les repères chiffrés qu'on emploie de nos jours pour désigner la longitude et la latitude.

Afin de mieux circonscrire la place et l'évolution des technologies dans les construits sociaux que sont les organisations, reprenons maintenant notre réflexion sur la syntaxe des artefacts. Notre travail se distingue de celui de Hutchins par le fait, notamment, que nous ne nous intéressons pas à l'évolution historique d'artefacts. Plutôt, nous voulons connaître comment ceux-ci s'intègrent dans une organisation. Nous nous interrogerons par ailleurs sur la nature des régularités dans un contexte autre que celui de la navigation marine. De plus, nous tenterons de circonscrire comment on peut appréhender les technologies informatiques grâce à la syntaxe des artefacts, dans le but de cerner leurs caractéristiques et la manière dont elles sont actualisées dans un contexte matériel et social en évolution.

${ }^{4}$ Notre traduction 


\section{Les artefacts et le travail en mutation : le cas d'un département de la comptabilité en milieu hospitalier}

Notre recherche empirique a pris la forme d'une étude de cas (Giroux, 2003) portant sur l'informatisation du travail de commis au service de la perception des comptes dans le département de comptabilité d'un centre hospitalier ${ }^{5}$. Ce service a pour mission d'assurer l'ouverture, le suivi et l'encaissement des comptes à recevoir. Notre étude porte sur deux commis, que nous nommons Dominique et Evelyne ${ }^{6}$, qui gèrent les comptes des patients hébergés aux soins prolongés.

De façon générale, les patients sont hospitalisés pour un problème de santé avant d'être transférés à une unité des soins prolongés. Avant l'informatisation, c'est à partir d'une carte comptable jaune d'un format de papier A5 environ, document communément appelé carte jaune par les employés, que les commis de la comptabilité assuraient le suivi du compte des patients. Ainsi, lorsque le patient change d'unité, sa carte jaune est transférée des commis aux hospitalisations régulières aux commis des soins de longue durée.

La carte jaune est un document sur lequel étaient inscrites une foule d'informations. Au recto, la carte était organisée grâce à une série de rubriques où le personnel notait l'identité du patient, sa localisation dans l'hôpital, le tarif de la chambre occupée, les renseignements financiers concernant le crédit et le débit ayant trait à la facturation et au paiement de la chambre occupée par le patient. À l'endos de la carte était imprimée la liste des types de chambres. Au moment d'intégrer une unité de soins prolongés, le patient choisit le type de chambre dans lequel il sera hébergé. Le patient cochait alors son choix sur la carte et validait celui-ci en signant. De plus, une série de lignes imprimées servaient aux commis, pour y noter les différentes étapes des démarches suivies pour connaître le type de chambre choisie par le patient hébergé aux soins de longue durée. Tout autre document concernant le traitement du dossier (formulaire ou reçu d'encaissement, état de compte, etc.) était agrafé à la carte jaune et entreposé dans un dossier papier.

Avec l'arrivée de la nouvelle technologie, l'information jadis rassemblée sur la carte jaune s'est reconfigurée dans une série de documents informatisés présentés, un à un, à l'écran de l'ordinateur. Les données sont ainsi organisées visuellement sur différentes pages

\footnotetext{
${ }^{5}$ Je remercie Isabelle Guibert qui a colligé les données.

${ }^{6}$ Le nom des participants a été modifié afin d'assurer leur confidentialité.
} 
d'écran qui font état: de l'identité du patient, de ses déplacements dans les chambres de l'hôpital, des sommes dues, des sommes payées et du transfert de sommes d'argent d'un compte à un autre lorsque le patient change d'unité d'hébergement. Le dossier papier regroupe maintenant les autres documents qui ne sont pas intégrés dans l'ordinateur.

Depuis l'informatisation, les commis inscrivent désormais les données contextuelles liées au compte d'un patient sur une fiche autocollante bleue de type Post-it, qu'elles nomment papier bleu et qu'elles accolent au dossier papier. Ces données, autrefois inscrites à l'endos de la carte jaune, les aident à se retrouver dans leurs démarches auprès des patients. Les commis ont développé leurs propres abréviations et codes, par exemple, en identifiant au marqueur les éléments qui leur semblent importants.

À partir de l'examen de nos données empiriques, on peut constater que les régularités suivent une logique transactionnelle selon laquelle diverses parties entrent en relation les unes avec les autres afin d'effectuer un échange de biens ou de services contre une rétribution financière. Les régularités regroupées dans les artefacts répondent à une série de questions liées aux caractéristiques d'une transaction: qui ? quoi ? combien ? quand ? Plus précisément, ces régularités permettent de rendre compte de l'identité des parties engagées dans la transaction ainsi que de la nature de ce qui est échangé et de la fréquence de ces échanges. Le tableau suivant illustre les dimensions de la transaction et les régularités qui y sont associées.

En analysant nos données, il est d'abord ressorti que les mêmes régularités étaient présentes dans les différents artefacts avant et après l'informatisation. Toutefois, la combinaison de celles-ci dans les artefacts varie depuis l'arrivée de la technologie. À cet égard, nous avons recueilli de nombreux commentaires auprès de nos commis, confrontées au changement technologique :

«En la regardant (la carte jaune), tu le savais tout de suite »;

«(depuis l'informatisation) Il faut toujours se promener d'un tableau à l'autre...»

En somme, alors que sur la carte jaune se juxtaposaient les dimensions des transactions, dans les documents informatiques, les régularités caractérisant le compte de chaque patient sont dispersées sur divers documents informatisés. 
Dossier : Conduire le changement organisationnel?

Sur la carte jaune, la présence simultanée de l'ensemble des renseignements sur les transactions financières entre le patient et l'hôpital permettait donc d'appréhender, d'un seul regard, tous les repères de la transaction. Elle offrait, par conséquent, un horizon d'action qui diffère des documents informatisés, lesquels ne permettent pas de connaître simultanément des renseignements tels que l'identification des parties engagées dans la transaction, le type de chambre, les échéances ainsi que les montants et dates des paiements reçus au compte du patient.

La nature et l'évolution des régularités inscrites dans les artefacts

\begin{tabular}{|c|c|c|}
\hline $\begin{array}{l}\text { Dimensions de } \\
\text { la transaction }\end{array}$ & Nature de l'information & $\begin{array}{c}\text { Sources des régularités } \\
\text { pour organiser } \\
\text { l'information }\end{array}$ \\
\hline Quoi & $\begin{array}{l}\text { Type de chambre : } \\
\text { - } \quad \text { privée } \\
\text { - } \quad \text { semi-privée } \\
\text { - } \quad \text { ou publique }\end{array}$ & $\begin{array}{l}\text { Disponibilité de } \\
\text { l'espace : norme } \\
\text { organisationnelle } \\
\text { Les } 3 \text { types de } \\
\text { chambres : norme } \\
\text { gouvernementale }\end{array}$ \\
\hline Combien & $\begin{array}{l}\text { Rétribution payée pour les } \\
\text { chambres, privées, semi- } \\
\text { privées ou publiques }\end{array}$ & $\begin{array}{c}\text { Les prix sont fixés par } \\
\text { des normes } \\
\text { gouvernementales }\end{array}$ \\
\hline Qui & $\begin{array}{l}\text { Rétribution acquittée par: } \\
-\quad \text { Gouvernement } \\
\text { - } \quad \text { Compagnie d'assurance } \\
\text { - } \quad \text { Patient }\end{array}$ & $\begin{array}{l}\text { Les parties qui sont } \\
\text { autorisées à payer la } \\
\text { chambre sont désignées } \\
\text { par des normes } \\
\text { gouvernementales }\end{array}$ \\
\hline Quand & $\begin{array}{l}\text { Rétribution } \\
\text { mensuellement } \\
\text { Tenue de livre et suivi des } \\
\text { comptes }\end{array}$ & $\begin{array}{l}\text { Selon la logique des } \\
\text { loyers : norme culturelle } \\
\text { Selon la logique } \\
\text { comptable : normes } \\
\text { professionnelles }\end{array}$ \\
\hline
\end{tabular}

Figure 1:

Les régularités associées aux différentes dimensions de la transaction 
Avant l'informatisation, la carte jaune découpait le processus comptable autour du patient comme unité de travail. Le patient gardait en effet toujours la même carte, peu importe l'aile d'hébergement où il se trouvait. Au service de la perception, les commis se transmettaient entre elles les cartes jaunes des patients qui changeaient d'unité d'hébergement.

Depuis l'informatisation, les documents produits par le logiciel sont découpés par cas, un cas désignant le séjour d'un patient dans une unité d'hébergement particulière. Lorsque le patient change d'unité, c'est-à-dire qu'il passe, par exemple, des soins actifs aux soins prolongés, une nouvelle série de documents informatisés est produite portant un nouveau numéro de cas. Ainsi, le séjour d'un patient est découpé en de multiples cas si celui-ci change plusieurs fois d'unité d'hébergement au cours de son hospitalisation.

Puisque les comptes peuvent être hiérarchisés selon différents critères, il est devenu possible d'observer un changement de l'ordre du «qui » au «quoi», soit du patient engagé dans la transaction financière au type de service offert, c'est-à-dire l'hébergement de ce patient dans différentes unités de l'hôpital. Cette nouvelle hiérarchie des données caractérisant l'artefact technologique a, selon nous, fortement contribué au sentiment de «perte du patient» exprimé par les commis au cours de notre étude : «la carte jaune les rendait plus humains (les malades) ».

Nous croyons que l'introduction du papier bleu collé au dossier du patient exerce l'importante fonction de réintroduire le patient dans le processus de travail des commis à l'hébergement. Le patient, autrefois matérialisé par le truchement de la carte jaune, l'est désormais par l'intermédiaire d'une fiche bleue et il continue ainsi d'occuper une place prédominante dans l'univers informationnel des commis.

\section{La syntaxe des artefacts : un concept pour expliquer les univers matériels et sociaux en mouvance}

À partir de notre recherche, nous avons pu observer que les régularités tirent leur origine dans les logiques organisationnelle, professionnelle et culturelle, tel qu'indiqué au tableau 1. À celles-ci s'ajoutent d'autres régularités que les travailleurs observés ont fait naître, par exemple, lors de la création du papier bleu. Ces régularités se distinguent de celles qui proviennent d'autres sources, de collectifs organisationnels ou professionnels, lesquels sont régis par des repères 


\section{Dossier : Conduire le changement organisationnel?}

institutionnalisés dont le choix a déjà fait l'objet de négociations dans un contexte autre que celui de notre étude (Strum \& Latour, 1987).

Ainsi, nous constatons que l'activité humaine prend forme à la jonction de différentes logiques dont les repères se matérialisent dans les artefacts. Plus particulièrement, notre étude illustre que la manière dont ces régularités s'articulent à même les artefacts habilite tout en contraignant ceux qui les manipulent. Nous avons observé que les différentes configurations d'une même série de régularités modifient le rapport qu'entretiennent les commis avec leur environnement de travail. D'un ensemble de transactions faciles à réaliser, appuyées par la carte jaune permettant de donner forme au patient, les commis sont passées à des documents informatisés qui forcent à une utilisation éclatée, tant en ce qui a trait aux différentes dimensions de la transaction qu'au séjour du patient à l'hôpital.

Le contexte de changement tel que nous l'avons étudié nous amène à nous interroger sur l'articulation entre la situation et l'artefact. Les exemples que nous venons de citer nous donnent à penser que l'artefact contribue à définir le cadre que met en œuvre l'employé pour appréhender une situation de travail. Ce rapport entre artefact et situation n'est cependant pas unidirectionnel. Nos données nous permettent en effet de constater que la situation contribue parfois à l'émergence de l'artefact. C'est le cas du papier bleu, créé par les commis pour faciliter la gestion des comptes des patients. Cet artefact a donc été conçu pour répondre aux besoins des commis dans la conduite de leurs tâches, dans le cadre d'un milieu de travail nouvellement informatisé. Il nous apparaît ainsi que la situation et les artefacts se constituent réciproquement à différents moments de leur évolution respective.

Néanmoins, la situation et les artefacts eux-mêmes se transforment sous l'effet des décisions humaines, lesquelles tentent de les circonscrire. Au moment de concevoir un artefact, qu'il soit émergent ou le fruit d'un long processus de planification, une série de questions se posent afin de désigner ce qui constitue une régularité, de choisir les régularités à intégrer et de définir comment juxtaposer ces dernières. Ces préoccupations, selon Suchman (1994), sont indéniablement liées à l'exercice du pouvoir et à l'implantation d'un système de discipline qui accompagnent toute forme de catégorisation. Nous croyons, à l'instar de Suchman, que les problèmes associés à la technologie découlent non seulement d'une volonté de discipliner les employés, mais aussi d'une méconnaissance du travail des personnes 
qui prennent les décisions, que celles-ci soient designers, membres de l'équipe de direction, ou simples exécutants.

Penser les technologies à partir de la syntaxe des artefacts nous aide ainsi à percevoir le changement comme une reconfiguration des logiques d'action, lesquelles prennent forme dans les interactions et les artefacts, qui eux-mêmes soit reproduisent d'anciennes façon de faire, soit voient nouvellement le jour au sein des membres de l'organisation. De manière plus générale, cette recherche nous amène à réfléchir plus en profondeur à la dimension organisante des artefacts, tant ceux qui meublent depuis des décennies nos espaces de travail que ceux que nous sommes appelés à créer de manière planifiée ou émergente.

\section{Bibliographie}

GIDDENS, Anthony. The Constitution of Society. Cambridge, MA: The Polity Press, 1984, 402 p.

GIROUX, Nicole. L'étude de cas. In : GIORDANO, Y. coord. Conduire un projet de recherche: une perspective qualitative. Paris: Éditions EMS, 2003, p. 42-84.

GROLEAU, Carole. Structuration, Situated Action and Distributed Cognition: Rethinking the Computerization of Organizations. Système d'Information et Management, 2002, vol.2, no 7, p. 13-36.

HUTCHINS, Edwin. Cognition in the Wild. Cambridge, MA: MIT Press, 1995, $381 \mathrm{p}$.

LATOUR, Bruno. La science en action, Paris, La Découverte, 1989, $450 \mathrm{p}$.

ORLIKOWSKI, Wanda. The Duality of Technology: Rethinking the Concept of Technology and Organizations. Organization Science, 1992, vol.3, no.3, p. 398-472.

ORLIKOWSKI, Wanda. Using Technology and Constituting Structures; A Practical lens for Studying Technology in Organizations. Organization Science, 2000, vol. 11, no. 4, p. 404-428.

PUTNAM, Linda, PACANOWSKI, Michael E.. Communication and Organizations; An Interpretive Approach. Newbury Park, Ca: Sage Publications, 1983, $303 \mathrm{p}$.

SIMONDON, Gilbert. Du mode d'existences des objets techniques. Paris, Montaigne, 1969, $256 \mathrm{p}$. 
Dossier : Conduire le changement organisationnel?

STRUM, Shirley C., LATOUR, Bruno. Redefining the Social Link : From Baboons to Humans. Social Science Information, 1987, vol. 26, no.4, p. 783-802.

SUCHMAN, Lucy. Do Categories Have Politics? The Language Action Perspective Reconsidered. Computer Supported Cooperative Work, 1994, Vol. 2, pp. 177-190.

TAYLOR, James R., VAN EVERY, Elizabeth J. The Emergent Organization :Communication as Its Site and Surface. Mahway, NJ: Lawrence Erlbaum Associates, 2000, 351 p. 
